\title{
An experience of hyperbaric oxygen and transurethral coagulation combination therapy for abnormal vessels and intractable macrohematuria due to pelvic arteriovenous malformations
}

\author{
Seiya Inoue ${ }^{1,2}$, Masashi Honda ${ }^{2^{*}}$, Shinji Hirano ${ }^{2}$, Noriya Yamaguchi², Bunya Kawamoto ${ }^{2}$, \\ Tsounapi Panagiota ${ }^{2}$, Katsuya Hikita ${ }^{2}$, Kuniyasu Muraoka ${ }^{2}$, Takehiro Sejima ${ }^{2}$, Chihiro \\ Takahashi $^{1}$, Atsushi Takenaka ${ }^{2}$ \\ ${ }^{1}$ Department of Urology, National Hospital Organization, Yonago Medical Center, Yonago, Japan; \\ ${ }^{2}$ Department of Urology, Tottori University School of Medicine, Yonago, Japan; \\ *Corresponding Author: honda@med.tottori-u.ac.jp
}

Received 6 August 2013; revised 5 September 2013; accepted 16 September 2013

Copyright (C) 2013 Seiya Inoue et al. This is an open access article distributed under the Creative Commons Attribution License, which permits unrestricted use, distribution, and reproduction in any medium, provided the original work is properly cited.

\begin{abstract}
A 78-year-old man was admitted to our hospital with a chief complaint of gross hematuria. The patient was diagnosed with pelvic arteriovenous malformations and repeated arterial embolization. A cystoscopy demonstrated that bladder trigone was covered by many clots. At the same time, bleeding mucosa at the right side of the bladder dome was confirmed. We administered hyperbaric oxygen therapy 7 days after admission. Macrohematuria recovered gradually and we then performed transurethral coagulation at the dome with failed mucosa 14 days after admission. Macrohematuria recovered completely and there was no recurrence during hospitalization. Thirty days after admission, we performed cystoscopy and found clear mucosa in the trigone. The patient was discharged 32 days after admission.
\end{abstract}

Keywords: Hyperbaric Oxygen Therapy; Macrohematuria; Pelvic Arteriovenous Malformations; Transurethral Coagulation

\section{INTRODUCTION}

Pelvic arteriovenous malformations (P-AVM) are rare, especially in males. Angiography is effective not only for the diagnosis, but also for embolization therapy of AVM in the pelvis; however, we sometimes have to repeat embolization many times because of continuous symptoms or insufficient efficacies. In this report, we attempted hyperbaric oxygen therapy (HBO) and transurethral coagulation (TUC) for abnormal vessels and persistent macrohematuria from P-AVM and showed good efficacies.

\section{CASE REPORT}

A 78-year-old man was admitted to our hospital with a chief complaint of gross hematuria. It started 2 weeks before his admission. He was diagnosed with P-AVM 7 years before his admission and bilateral iliac artery embolization was performed a few times in another hospital. He used gabapentin for the continuous supurapubic dull pain that had continued for 20 years. There was no history of previous trauma or abdominal surgery. His physical examination was unremarkable. Laboratory data showed urinalysis with many red blood cells per high power field. Prothrombin time, partial thromboplastin time, and platelet count were normal. Ultrasonography demonstrated a hypoechoic region over the urinary bladder. An abdominal-pelvic computed tomography (CT) scan demonstrated a large mass consisting of dilatedtortuous abnormal vessels in the pelvis that was adherent to the anterior wall of the bladder and extended to the bottom of bladder. An enhanced CT scan was performed and we observed abnormal vessel invasion near the trigone (Figure 1). Cystoscopy revealed bleeding mucosa at the dome of the bladder wall (Figure 2). In addition, the bladder trigone was covered by many clots where the enhanced CT scan revealed abnormal vessel invasion. We diagnosed macrohematuria from the dome of the urinary bladder and abnormal vessels in the trigone due 
to P-AVM. We treated conservatively with tranexamic acid and carbazochrome intravenously; then, started HBO with 2.0 ATA for $60 \mathrm{~min} /$ day $\times 5$ days/week for four weeks. Macrohematuria ameliorated slowly. Thus, we performed TUC 7 days after starting HBO. In this surgery, bladder trigone was reddish and full of abnormal microvessels (Figure 3). The dome still exhibited slight bleeding from failed mucosa. TUC was performed at the dome of bladder mucosa. After that, the patient exhibited no recurrence of macrohematuria and an uneventful postoperative course. On day 30, cystoscopy revealed clear manifestation in the bladder dome. Surprisingly, most of the abnormal vessels had disappeared in the trigone (Figure 4). He was discharged 32 days after being admitted. The patient had no evidence of recurrence through 1 month of follow-up.

\section{DISCUSSION}

AVM is uncommon vascular lesions built by multiple abnormal communications between arterial and venous systems without a normal intervening capillary network [1]. AVM is generally agreed that the majority of lesions are congenital, with a few that being acquired

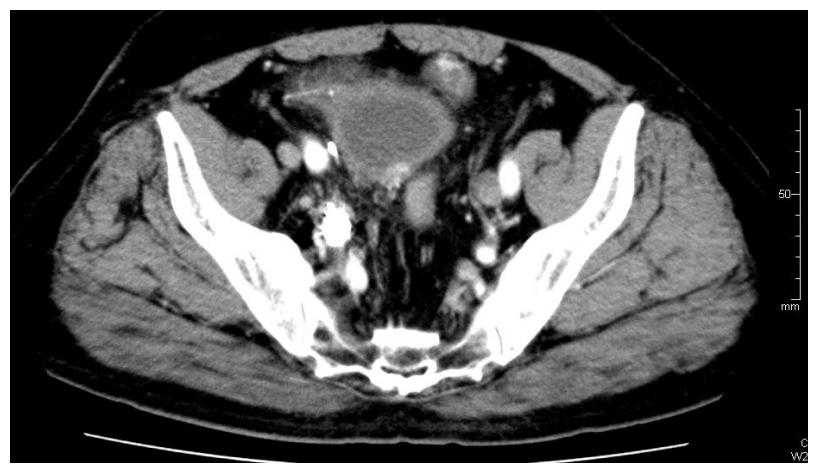

Figure 1. A CT scan showed a large pelvic arteriovenous malformation. Enhanced vessels are observed at the trigone of the urinary bladder (arrow).

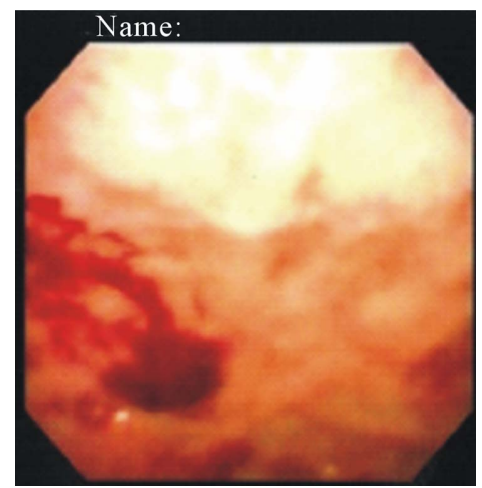

Figure 2. A Cystoscopy demonstrated a mucosal hemorrhage at the right side of the bladder dome.

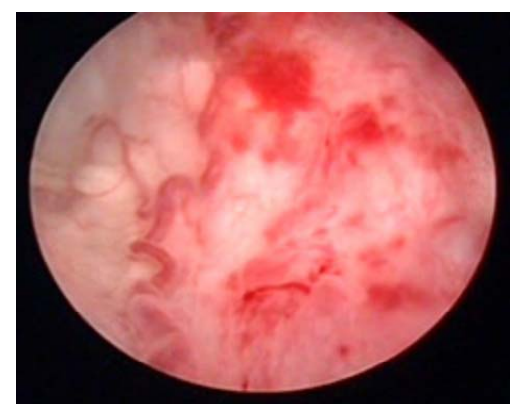

Figure 3. A cystoscopy showed reddish mucosa and many abnormal vessels in the bladder trigone 14 days after admission.

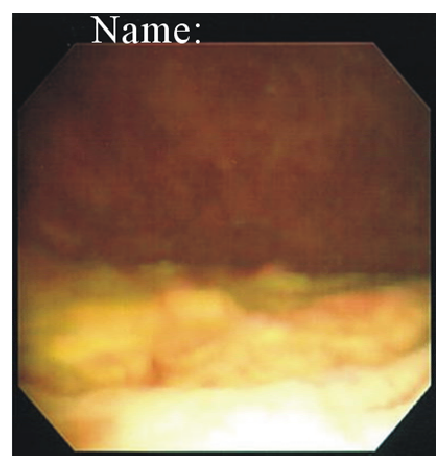

Figure 4. A cystoscopy showed a normal mucosa in the bladder trigone 30 days after admission.

after trauma or abdominal surgery. AVM in pelvic space is rare. Rosenberg et al. reported the first case of AVM of the bladder [2]. Presenting symptoms of P-AVM include pelvic discomfort and pain, rectal pain, genitourinary complaints including hematuria, hydronephrosis, hemospermia, and erectile dysfunction. However, most are asymptomatic. Physical examinations can reveal a pulsatile mass, and a harsh noise can be heard. When $\mathrm{P}-\mathrm{AVM}$ are suspected, an angiogram and pelvic CT or magnetic resonance imaging (MRI) are essential to delineate the extent of the disease. Diagnosis is confirmed by angiography. CT and MRI are useful to demonstrate the vascular etiology of the lesion, to evaluate its extension and involvement of adjacent structures, and to demonstrate the feeding vessels supplying the malformation and draining veins [3]. Ultrasonography reveals nonspecific hypoechogenic regions. However, it cannot differentiate P-AVM from a cyst or an abscess. Colored Doppler ultrasonography is useful to detect color images of the vascular structure. Treatments consist of conventional therapy, arterial embolization, or surgical excision including transurethral resection, tumor removal, or ligation of the feeding vessels [1]. If symptoms are relatively mild or the tumor is not growing, palliative therapy can be selected. Recently, arterial embolization is the most 
common method used as the first line of treatment; however, it often fails. The most suitable indication of surgical treatment is still controversial; however, a lower number of collaterals or a small tumor volume is easy to resolve with surgical treatment. HBO therapy is indicated for patients with hemorrhagic cystitis caused by radiation, intractable interstitial cystitis, or cyclophosphamide-induced hemorrhagic cystitis. Nakada et al. demonstrated that essential macrohematuria patients treated with HBO (2.0 ATA for 30 days) had a significantly superior recovery than those without any treatment. Moreover, the time period of recovery is shorter than that of non-treated patients [4]. It has been demonstrated that $\mathrm{HBO}$ is associated with vasoconstriction in the systemic circulation and to a lesser extent in the coronary vasculature [5]. Activation of plasma kinins results in vasodilation, edema formation, or hypotension. Animals treated in the hyperbaric chamber at a pressure of 2.5 ATA saturated with $100 \%$ oxygen showed low levels of plasma kinins [6]. Such treatment presumably results in vasoconstriction and contributes to decrease in hematuria. The biological mechanism of $\mathrm{HBO}$ originated from hypersaturating oxygen dissolved in the plasma due to the treatment. Subsequently, HBO treatment stimulates leukocyte function characterized by phagocytosis, producing growth factors that enhance angiogenesis [7]. HBO therapy is well tolerated, and adverse effects of $\mathrm{HBO}$ are unusual, as visual disturbances, eustachian tube dysfunction, and claustrophobia are rare [8]. To our knowledge, there have been no reports about the use of HBO and TUC combination therapy for P-AVM. The present case showed the difficulties in treating macrohematuria in spite of repeated arterial embolization. We performed HBO and TUC combination therapy for persistent macrohematuria, and confirmed a recovery of macrohematuria and reduction of abnormal vessels at the trigone. During HBO therapy, the patient felt ear fullness that resolved itself automatically. We supposed that under a high pressure of oxygen, angiogenesis progressed and abnormal vessels and damaged mucosa recovered. As this is a case report of only one patient, our data pro- vide the possibility of another treatment option for intractable macrohematuria and abnormal vessels patients who do not respond to conventional therapies. Careful observations are required.

\section{REFERENCES}

[1] Game, X., Berlizot, P., Hassan, T., Joffre, F., Chokairi, S., Houlgatte, A. and Rischmann, P. (2002) Congenital pelvic arteriovenous malformation in male patients: A rare case of urological symptoms and role of embolization. European Urology, 42, 407-412. http://dx.doi.org/10.1016/S0302-2838(02)00355-X

[2] Rosenberg, J., Golimbu, M., Suarez, J. and Morales, P. (1973) Congenital arteriovenous malformation of the bladder. Journal of Urology, 109, 605-608.

[3] Jacobowitz, G.R., Rosen, R.J., Rockman, C.B., Nalbandian, M., Hofstee, D.J., Fioole, B., Adelman, M.A., Lamparello, P.J., Gagne, P. and Riles, T.S. (2001) Transcatherter embolization of complex pelvic vascular malformations: Results and long-term follow-up. Journal of Vascular Surgery, 33, 51-55.

[4] Nakada, T., Sasagawa, I., Koike, H., Furuta, T., Katayama, K., Ota, K., Chikenji, M., Matsushita, T. and Saito, H. (1989) Effect of hyperbaric oxygen therapy on essential haematuria. International Urology and Nephrology, 21, 3-8.

[5] Mogelson, S., Davidson, J., Sobel, B.E. and Roberts, R. (1981) The effect of hyperbaric oxygen on infarct size in the conscious animal. European Journal of Cardiology, 12, 135-146.

[6] Sumida, S. and Yagi, H. (1981) Experimental study on the inhibition of kinin release in endotoxin shock by glutathione, proteinase inhibitors, hydrocortisone and hyperbaric oxygen. Japanese Circulation Journal, 45, 13641368.

[7] Hader, J.E., Marzella, L., Myers, R.A., Jacobs, S.C. and Naslund, M.J. (1993) Hyperbaric oxygen treatment for experimental cyclophosphamide-induced hemorrhagic cystitis. Journal of Urology, 149, 1617-1621.

[8] Capelli-Schellpferffer, M. and Gerber, G.S. (1999) The use of hyperbaric oxygen in urology. Journal of Urology, 162, 647-654. 